\section{Desenvolvimento de um \\ questionário de freqüência \\ alimentar para avaliação do \\ consumo alimentar de crianças de \\ 2 a 5 anos de idade}

\section{Development of a food frequency questionnaire for children aged 2 to 5 years}

Ana Carolina Almada Colucci

Departamento de Nutrição

Faculdade de Saúde Pública

Universidade de São Paulo

Av. Dr. Arnaldo, 715 - Cerqueira César

01246-904 - São Paulo - SP

colucci@usp.br

\section{Sonia Tucunduva Philippi}

Departamento de Nutrição

Faculdade de Saúde Pública

Universidade de São Paulo

Betzabeth Slater

Departamento de Nutrição

Faculdade de Saúde Pública

Universidade de São Paulo

\section{Resumo}

Devido ao crescente interesse sobre a possível relação entre a alimentação de crianças e adolescentes e doenças na idade adulta, a dieta de indivíduos jovens tem sido pesquisada. Este trabalho teve por objetivo desenvolver um questionário de freqüência alimentar para avaliar a dieta habitual de crianças de 2 a 5 anos de idade (QFAC). Foram identificados os alimentos mais representativos para o consumo de energia - carboidrato, proteína, lipídio, retinol, vitamina $\mathrm{C}$, cálcio e ferro - a partir de recordatórios de $24 \mathrm{~h}$ de crianças de 2 a 5 anos do município de São Paulo. No QFAC foram definidas porções médias ou medianas, o tempo precedente foi estipulado em 6 meses e a freqüência de consumo apresentada em 7 categorias. O QFAC apresentou-se como um instrumento adequado para a avaliação da dieta habitual de crianças de 2 a 5 anos de idade, possibilitando a investigação das características da alimentação habitual deste grupo e o estabelecimento das possíveis relações entre a dieta e o estado nutricional.

Palavras-chave: Questionário de Freqüência Alimentar. Consumo alimentar. Alimentação infantil. 
This study was conducted to design a food frequency questionnaire for assessing the regular diets of children aged 2 to 5 years. It identified the most important sources of total calories, carbohydrate, proteins, total fat, retinol, vitamin C, calcium and iron from $24 \mathrm{~h}$ dietary recalls applied to a random population sample aged 2 to 5 years in the city of São Paulo. Average or median portions were defined. The FFQ investigated the frequency of children's consumption of 57 items over the previous six months. Seven categories of food frequency were defined. The FFQ proved to be useful in epidemiological studies of children's intake over extended periods, making it possible to identify the dietary habits of this group and to evaluate the association between diet and nutritional status.

Keywords: Food Frequency Questionnaire. Food intake. Infant nutrition.

\section{Introdução}

Há evidências concretas de que algumas doenças típicas do adulto (obesidade, doenças cardíacas, câncer e osteoporose) são processos patológicos que refletem a exposição acumulativa a fatores de risco, dentre eles a dieta, em diferentes fases da vida. Durante a infância e a adolescência, a alimentação, ao mesmo tempo em que é importante para o crescimento e desenvolvimento, pode também representar um dos principais fatores de prevenção de algumas doenças na idade adulta ${ }^{1,2}$.

Diante desta realidade, tem-se observado grande interesse pelo monitoramento de indicadores dietéticos para crianças e adolescentes, pois a partir destes é possível a identificação e compreensão das inter-relações entre as escolhas alimentares e o estado de saúde ${ }^{1-2}$.

Em vista da grande variação diária da alimentação e da rápida mudança dos hábitos alimentares infantis, o desenvolvimento de instrumentos capazes de medir com exatidão a dieta habitual das crianças apresentase como um importante desafio metodológico. Além disso, deve-se considerar a limitada capacidade cognitiva deste grupo ${ }^{4}, \mathrm{o}$ que faz com que a informação dietética deva ser obtida com os adultos responsáveis pela criança, em casa ou na escola ${ }^{4}$.

Dentre os métodos mais utilizados para mensurar o consumo alimentar de crianças, merece destaque o Questionário de Freqüência Alimentar (QFA), amplamente empregado na epidemiologia nutricional por possibilitar a classificação de grupos populacionais de acordo com o seu consumo alimentar habitual, a identificação de indivíduos com padrões extremos de consumo e o monitoramento de tendências nos comportamentos alimentares ao longo do tempo ${ }^{5-7}$.

Na literatura internacional, várias pesquisas foram conduzidas nos últimos anos com o objetivo de avaliar com confiabilidade a dieta consumida por crianças e adolescentes, utilizando o QFA ${ }^{8-11}$. No Brasil, a literatura disponível ainda é escassa, sendo que a maior parte dos trabalhos foram realizados 
com adultos ${ }^{12}$; somente o estudo realizado por Slater et al. ${ }^{13}$ teve como objetivo desenvolver um QFA para adolescentes.

Considerando a inexistência de trabalhos nacionais utilizando o QFA para a avaliação do consumo alimentar habitual das crianças e a necessidade de aperfeiçoar e agilizar a avaliação da dieta consumida por este grupo, foi desenvolvido um Questionário de Freqüência Alimentar para avaliação do consumo alimentar de Crianças (QFAC) de 2 a 5 anos de idade, residentes no município de São Paulo.

\section{Casuística e Métodos}

\section{População de estudo}

Para a elaboração da estrutura do QFAC, foram utilizados os dados da pesquisa "A trajetória da saúde infantil como medida de desenvolvimento social: o caso da cidade de São Paulo ao longo de cinco décadas”, realizada pelo Núcleo de Pesquisas Epidemiológicas em Nutrição e Saúde - NUPENS, da Faculdade de Saúde Pública da Universidade de São Paulo. Neste estudo, nutricionistas treinados realizaram entrevistas com as mães e/ou responsáveis pelas crianças. Foi aplicado inquérito Recordatório de 24 horas (R24h) a todas as crianças menores de dois anos e em uma a cada três crianças com idade entre dois e cinco anos. ${ }^{14}$

Do total de 718 crianças, foram avaliados neste trabalho somente os R24h de 207 crianças de 2 a 5 anos de idade, de ambos os sexos, pelo fato de que a partir dos 2 anos a criança já consome alimentação variada, com diferentes consistências e formas de preparo, semelhante à alimentação da família.

\section{Desenvolvimento do QFAC}

Identificação e agrupamento dos alimentos

A partir dos alimentos consumidos pelas crianças e presentes nos R24h elaborou-se uma lista com 389 alimentos, com informa- ções detalhadas sobre especificidade, forma de preparo, marca comercial e conteúdo de nutrientes.

Posteriormente, os alimentos foram agrupados em 88 itens homogêneos segundo características e perfil de nutriente, consultando-se tabelas de composição centesimal $^{15,16}$ e o "software" Virtual Nutri1 ${ }^{17}$, versão 1.0, para obtenção do valor nutritivo.

\section{Listas de alimentos}

Após o agrupamento, foram elaboradas as listas com os alimentos que mais contribuíam para a ingestão de energia, carboidrato, lipídio, proteína, retinol, vitamina C, cálcio e ferro na dieta das crianças. As listas foram construídas aplicando-se técnica de análise estatística proposta por Block et al. ${ }^{18}$ e Howe et al. ${ }^{19}$, sendo o nutriente total consumido pela população estimado pelo somatório do nutriente nas porções dos alimentos referidos. Desta forma, a contribuição percentual de cada alimento em particular $i$ foi dada pela Equação 1 .

A partir das listas de contribuição para cada alimento, foi estabelecido o ponto de corte de $80 \%$ de contribuição ao total do nutriente, como critério de inclusão dos alimentos no $\mathrm{QFAC}^{2,18,20}$. Para o retinol e a vitamina $\mathrm{C}$, os pontos de corte adotados foram, respectivamente, $55 \%$ e $60 \%$. A lista final do QFAC apresentou 57 itens alimentares.

\section{Tamanho das porções}

A definição do tamanho das porções para cada alimento foi obtida nos R24h, pelo cálculo da quantidade média e mediana em gramas consumida pelas crianças. A maioria das porções incluídas no QFAC expressa o consumo médio em gramas com exceção de 15 alimentos que apresentaram diferenças significativas entre os sexos, para os quais foi adotada a porção mediana (em gramas). Após a definição da quantidade em gramas a ser inserida no QFA, foi realizada a transformação destas estimativas em medidas caseiras, para facilitar a compreensão e por

Conteúdo do nutriente (p.ex.vitamina $C$ ) fornecido pelo alimento $i$ Total do nutriente fornecido por todos os alimentos 
refletirem a forma cotidiana de consumo das crianças.

\section{Determinação do tempo precedente}

O QFAC foi desenvolvido para avaliar o consumo alimentar habitual de crianças de 2 a 5 anos de idade durante os 6 meses precedentes à sua aplicação. $\mathrm{O}$ tempo precedente utilizado em QFA desenvolvidos para crianças, em trabalhos existentes na literatura $^{8-11}$, variou desde uma semana ${ }^{10}$ até um ano precedentes ${ }^{21}$, em função do grupo e do fator dietético estudado.

No QFAC, a adoção de 6 meses precedentes, como também fizeram Stein et al. ${ }^{11}$ e Basch et al..$^{22}$ permite supor que seja possível a avaliação global da dieta, considerando a variabilidade nas preferências alimentares das crianças.

\section{Freqüência de consumo dos alimentos}

Para cada alimento, foram estabelecidas questões simples com respostas múltiplas $\mathrm{e}$ fechadas. Foram definidas sete categorias: nunca, menos de 1 vez por mês, 1 a 3 vezes por mês, 1 vez por semana, 2 a 4 vezes por semana, 1 vez por dia e 2 ou mais vezes por dia, salientando que todos os alimentos apresentaram as mesmas opções ${ }^{13}$. Foi ainda incluído um espaço aberto para possíveis alimentos não existentes no QFAC.

\section{Resultados}

Na Tabela 1 estão apresentados, em ordem alfabética, os 48 alimentos selecionados a partir das listas e incluídos no QFAC, e sua contribuição percentual ao consumo total de calorias, carboidratos, proteínas, lipídios, cálcio e vitamina C. Nesta Tabela, o traço (-) apresentado em alguns alimentos indica que os mesmos não contribuíram de forma representativa para o consumo total do nutriente descrito. Observa-se que o leite fluido se apresenta como o alimento que mais contribuiu para o consumo total de energia, proteínas, lipídios e cálcio, devido à sua enorme representatividade e elevada freqüência de consumo na dieta daquelas crianças. Com relação aos carboidratos, os ali- mentos que mais contribuíram percentualmente foram o açúcar refinado e o arroz branco cozido, representando quase $30 \%$ do total consumido pela população. Para a vitamina C, como esperado, as frutas, verduras e legumes predominaram, sendo que o consumo de laranja ou suco de laranja contribuiu com aproximadamente $20 \%$.

A Tabela 2 apresenta a descrição de alguns itens alimentares e respectivas porções (em gramas e medidas caseiras) que integram o QFAC.

Apesar de não selecionados a partir das listas de contribuição, sete itens alimentares foram incluídos no QFAC, considerando, pela observação prática, que estes alimentos estão atualmente presentes na dieta habitual das crianças: chuchu, mandioquinha, requeijão, pizza, sanduíche (misto, hambúrguer), chá e água. Foram também adicionados exemplos das possíveis formas de preparo para determinado item alimentar ou foi utilizada a marca comercial para melhor identificação.

Finalmente, após a definição de todos os parâmetros, o instrumento desenvolvido apresentou 57 itens alimentares, divididos em 10 categorias, adotando-se em alguns casos a nomenclatura utilizada por Philippi et al. ${ }^{23}$ para a Pirâmide Alimentar: Arroz, pão, massa, batata; Feijão; Verduras e Legumes; Frutas; Carnes e ovos; Leite, queijo, iogurte; Açúcar, doces e salgadinhos; Salgados e preparações; Bebidas e Outros (Figura 1).

\section{Discussão}

O conhecimento das relações entre a dieta das crianças e adolescentes e as enfermidades na vida adulta torna-se um estímulo para a avaliação do consumo alimentar de indivíduos jovens, com ênfase na descrição das práticas alimentares adotadas, no monitoramento de tendências a longo prazo e, finalmente, no delineamento de estudos de intervenção.

Assim, por ser imprescindível a utilização de instrumentos adequados para o conhecimento da dieta habitual de crianças, foi desenvolvido o QFAC, como um instru- 
Tabela 1 - Lista de alimentos incluídos no QFAC e sua contribuição percentual para o total de calorias, carboidratos, proteínas, lipídios, cálcio e vitamina C da dieta de crianças de 2 a 5 anos de idade da cidade de São Paulo, 1995-1996. Table 1 - List of foods included in the FFQ and their contribution to calories, carbohydrates, protein, total fat, calcium and vitamin Cin the diet of 2 to 5 year old children: data from São Paulo, 1995-1996.

\begin{tabular}{|c|c|c|c|c|c|c|}
\hline Alimentos & $\begin{array}{c}\text { Calorias } \\
\text { (\%) }\end{array}$ & $\begin{array}{c}\text { Carboidratos } \\
\text { (\%) }\end{array}$ & $\begin{array}{l}\text { Proteínas } \\
\text { (\%) }\end{array}$ & $\begin{array}{l}\text { Lipídios } \\
\text { (\%) }\end{array}$ & $\begin{array}{l}\text { Cálcio } \\
(\%)\end{array}$ & $\begin{array}{c}\text { Vitamina C } \\
(\%)\end{array}$ \\
\hline Achocolatados em pó & 2,35 & 3,87 & $-*$ & - & - & 7,86 \\
\hline Açúcar refinado & 7,62 & 14,17 & - & - & - & - \\
\hline Alface, acelga, agrião & - & - & - & - & - & 1,17 \\
\hline Arroz branco cozido & 9,11 & 13,79 & 4,67 & 2,49 & 3,16 & 1,06 \\
\hline Banana & - & 1,46 & - & - & - & 1,64 \\
\hline Batata cozida & - & - & - & - & - & 1,61 \\
\hline Batata frita & - & - & - & 1,20 & - & 2,00 \\
\hline Bebida láctea, iogurtes & 1,10 & 1,47 & 0,98 & - & 1,44 & - \\
\hline Bife & 3,34 & - & 6,55 & 6,34 & - & - \\
\hline Biscoito sem recheio & 3,70 & 4,25 & 1,84 & 4,03 & - & - \\
\hline Biscoitos recheados & 2,37 & 2,44 & & 2,93 & - & - \\
\hline Bolo comum, chocolate, etc & 2,23 & 2,52 & 1,12 & 2,26 & 6,93 & - \\
\hline Café com açúcar & 0,88 & 1,85 & - & - & - & - \\
\hline Caju, goiaba & - & - & - & - & - & 1,24 \\
\hline Carne bovina (assada, refogada, etc) & 4,02 & - & 13,19 & 6,19 & - & 1,00 \\
\hline Cereal matinal & - & - & - & - & - & - \\
\hline Chocolate, bombons & 1,15 & 1,00 & - & 1,67 & - & - \\
\hline Doce de frutas, doce de leite, etc & - & - & - & - & - & - \\
\hline Embutidos (lingüiça, salsicha, etc) & 2,19 & - & 2,87 & 5,12 & - & - \\
\hline Espessantes & 2,38 & 2,47 & 0,96 & - & 3,92 & 2,25 \\
\hline Extrato de tomate, molho de tomate & - & - & - & - & - & 0,80 \\
\hline Feijão & 3,03 & 2,93 & 4,64 & 2,57 & 2,10 & - \\
\hline Fígado bovino & - & - & - & - & - & 0,84 \\
\hline Frango (frito, grelhado, assado, cozido) & 2,06 & - & 6,97 & 2,42 & - & - \\
\hline Fubá, polenta sem molho & - & - & - & - & - & - \\
\hline Laranja, suco de laranja & 1,39 & 2,46 & - & - & - & 18,85 \\
\hline Leite em pó integral & 6,17 & 3,53 & 8,51 & 10,41 & 19,24 & 2,95 \\
\hline Leite fermentado & - & - & - & - & 1,08 & - \\
\hline Leite fluido & 11,97 & 6,69 & 17,73 & 18,70 & 38,38 & 6,06 \\
\hline Leite materno & - & - & - & - & - & - \\
\hline Maçã/pêra & - & - & - & - & - & 0,87 \\
\hline Macarrão (cozido, ao sugo) & 1,13 & 1,67 & 1,06 & - & - & 1,67 \\
\hline Macarrão instantâneo & 0,90 & - & - & 1,20 & - & - \\
\hline Mamão & - & - & - & - & - & 2,65 \\
\hline Margarina/manteiga & 1,81 & - & - & 5,90 & - & - \\
\hline Ovo frito, pochê, omelete & 1,28 & - & 2,20 & 2,51 & - & - \\
\hline Pão (francês, forma, bisnaguinha) & 4,56 & 6,57 & 3,91 & 1,19 & 3,23 & - \\
\hline Peixe & - & - & 1,32 & - & - & - \\
\hline Queijo parmesão, prato & - & - & - & 1,30 & - & - \\
\hline Refrigerante & 1,73 & 3,37 & - & - & - & - \\
\hline Salgadinhos, batata chips & 2,34 & 1,71 & 1,03 & 1,60 & - & 1,84 \\
\hline Salgados (bolinha de queijo, pastel, etc) & - & - & - & - & 1,31 & - \\
\hline Sopa infantil com carne & - & - & 1,32 & - & - & 1,77 \\
\hline Sopa infantil sem carne & - & - & - & - & - & - \\
\hline Suco artificial em pó & - & 1,16 & - & - & - & - \\
\hline Suco de frutas & - & 1,01 & - & - & - & 1,46 \\
\hline Tomate & - & - & - & - & - & 1,15 \\
\hline
\end{tabular}

* O alimento não contribuiu de forma representativa ao consumo do nutriente. / ${ }^{*}$ The food item does not contribute significantly to the consumption of the nutrient. 
Tabela 2 - Itens alimentares e respectivas porções que integram o Questionário de Freqüência Alimentar para Crianças (QFAC)

Table 2 - Food items and size of portions of the Food Frequency Questionnaire for children from 2 to 5 years of age.

\begin{tabular}{|c|c|c|}
\hline Item alimentar & Medida caseira & Peso médio (g) \\
\hline Acelga/repolho/couve & 1 colher de sopa & $15,5^{*}$ \\
\hline Achocolatados em pó (Nescau ${ }^{\oplus}$, Toddy $\left.{ }^{\oplus}\right)$ & $11 / 2$ colher de sobremesa & 13,0 \\
\hline Açúcar & 1 1/2 colher de sobremesa & 21,0 \\
\hline Alface & 2 folhas & $15,5^{*}$ \\
\hline Arroz cozido & 3 colheres de sopa & 82,0 \\
\hline Banana & 1 unidade & 86,0 \\
\hline Batata cozida/purê & 1 colher de servir & 51,0 \\
\hline Batata frita & 1 escumadeira & 103,0 \\
\hline Bife & 1 unidade & 76,0 \\
\hline Biscoitos com recheio (chocolate, waffer) & 3 unidades & 39,0 \\
\hline Biscoitos sem recheio (maisena, maria, leite,água e sal) & 3 ou 4 unidades & $19,5^{*}$ \\
\hline Bolo comum/chocolate & 1 fatia & 83,0 \\
\hline Carne cozida (panela, moída) & $1 / 2$ fatia/3 colheres de sopa & 54,0 \\
\hline Cenoura & $1 / 2$ colher de servir & $15,0^{*}$ \\
\hline Chocolate/bombom & 1 unidade & 29,0 \\
\hline Danoninho ${ }^{\circ}$ Chambinho $^{\circ}$ & 1 pote & 51,0 \\
\hline Espessantes (Maizena ${ }^{\circ}$, Farinha Láctea ${ }^{\circ}$, Mucilon ${ }^{\circ}$, Cremogema ${ }^{\circ}$ ) & 1 ou 2 colheres de sopa & 19,0 \\
\hline Feijão & $1 / 2$ concha & $52,0^{*}$ \\
\hline Frango (cozido, frito, grelhado, assado) & 1 pedaço/1 unidade & 62,0 \\
\hline logurte de frutas & 1 pote & 138,0 \\
\hline Laranja & 1 unidade & 128,0 \\
\hline Leite fermentado tipo Yakult ${ }^{\circ}$, Chamyto ${ }^{\circ}$ & 1 pote & 96,0 \\
\hline Leite fluido integral/leite em pó integral diluído & 1 xícara & 184,0 \\
\hline Lingüiça/salsicha & $1 / 2$ gomo/1 unidade & $31,0^{*}$ \\
\hline Maçã/pêra & 1 unidade & 93,0 \\
\hline Macarrão cozido/ao sugo & 1 escumadeira & 72,0 \\
\hline Mamão & 1 fatia & 90,0 \\
\hline Margarina/manteiga & 1 colher de chá & 7,0 \\
\hline Pão francês/forma/bisnaguinha & $1 / 2$ unidade/ 1 fatia/1 unidade & $25,0^{*}$ \\
\hline Queijo prato/mussarela & 1 fatia & 14,0 \\
\hline Refrigerante & $1 / 2$ соро & 170,0 \\
\hline Salgadinho/batata chips & 1 pacote pequeno & 55,0 \\
\hline Salgados (pão de queijo, pastel, coxinha, esfiha) & 1 unidade pequena & 58,0 \\
\hline Sopa com carne & $1 / 2$ prato & $120,0^{*}$ \\
\hline Sopa sem carne & $1 / 2$ prato & 95,0 \\
\hline Suco artificial (tipo Tang ${ }^{\oplus}$ ) & $1 / 2$ соро & 164,0 \\
\hline Suco de laranja & $1 / 2$ copo & 128,0 \\
\hline Suco de outras frutas (maracujá, abacaxi) & $1 / 2$ copo & 116,0 \\
\hline Tomate & 3 fatias & 50,0 \\
\hline
\end{tabular}

* valor mediano /* median

mento capaz de descrever por estratos ou níveis de consumo as possíveis relações entre dieta e doença.

Em nosso meio, devido à inexistência de QFA especificamente desenvolvido para crianças, poder-se-ia supor que algumas difi- culdades operacionais, tais como: tempo dispendido, dificuldades no planejamento metodológico e análises estatísticas aprofundadas, apresentam-se como obstáculos aos pesquisadores.

A identificação inicial dos alimentos con- 
Assinale com um $\mathrm{X}$ a quantidade de cada alimento que a criança consumiu habitualmente durante os últimos 6 meses.

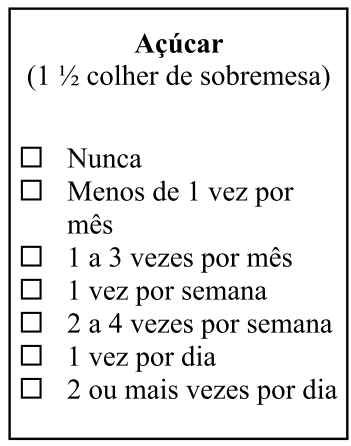

\begin{tabular}{|ll|}
\hline \multicolumn{1}{|c|}{$\begin{array}{c}\text { Chocolate/bombom } \\
\text { (1 unidade) }\end{array}$} \\
$\square \quad$ Nunca \\
$\square \quad$ Menos de 1 vez por \\
mês \\
$\square \quad 1$ a 3 vezes por mês \\
$\square \quad 1$ vez por semana \\
$\square \quad 2$ a 4 vezes por semana \\
$\square \quad 1$ vez por dia \\
$\square \quad 2$ ou mais vezes por dia \\
\hline
\end{tabular}

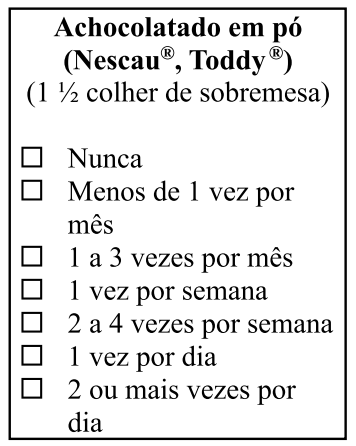

\begin{tabular}{|ll|}
\hline \multicolumn{1}{|c|}{$\begin{array}{c}\text { Bolo comum/chocolate } \\
\text { (1 fatia) }\end{array}$} \\
$\square \quad$ Nunca \\
$\square \quad$ Menos de 1 vez por \\
mês \\
$\square \quad 1$ a 3 vezes por mês \\
$\square$ & 1 vez por semana \\
$\square$ & 2 a 4 vezes por semana \\
$\square$ & 1 vez por dia \\
$\square$ & 2 ou mais vezes por \\
dia
\end{tabular}

Figura 1 - Estrutura do Questionário Questionário de Freqüência Alimentar para Crianças (QFAC).

Figure 1 - Structure of the Food Frequency Questionnaire for children from 2 to 5 years of age.

sumidos pelas crianças de 2 a 5 anos de idade foi realizada com a finalidade de garantir que o QFAC representasse os alimentos pertencentes à dieta habitual deste grupo.

Estão descritas na literatura diferentes estratégias para obtenção dos alimentos a serem inseridos em um QFA, como a identificação dos alimentos com base no conteúdo do nutriente (ex: fígado por ser rico em ferro) e a seleção baseada na orientação de um nutricionista experiente ${ }^{5}$ ou ainda a análise múltipla "stepwise" para cada nutriente, considerando como variável dependente a ingestão total do nutriente e como variáveis independentes os alimentos ${ }^{20}$. Tais abordagens são válidas e já foram utilizadas por alguns autores, porém é elevado o risco de exclusão de alimentos representativos ao consumo alimentar da população.

A escolha pela aplicação da análise estatística proposta por Block et al. ${ }^{18} \mathrm{e}$ utilizada por Slater et al. ${ }^{13}$ minimizou a possibilidade de omissão de algum alimento importante, dado que os alimentos incluídos refletiam o conteúdo do nutriente, a freqüência de consumo e a porção de referência consumida pelas crianças.

Para a maioria dos nutrientes avaliados, foi adotado o ponto de corte de $80 \%$ para inclusão dos alimentos no QFAC. Com o objetivo de evitar listas extensas que inviabilizariam a aplicação do questionário, para o retinol e a vitamina $\mathrm{C}$ foram adotados os pontos de corte de $55 \%$ e $60 \%$, respectivamente.

A lista dos alimentos do QFAC foi comparada com os itens citados em pesquisa realizada por Galleazzi et al. ${ }^{24} \mathrm{em}$ cinco cidades brasileiras. Apesar de, neste estudo, estar descrito o consumo aparente de alimentos por indivíduos de diferentes faixas etárias, tal comparação demonstrou que, do total de 16 alimentos citados como os mais consumidos pela população, 14 alimentos 
estavam presentes no QFAC. Os únicos itens não incluídos no instrumento foram a carne suína e a farinha de milho, alimentos realmente pouco consumidos por crianças de 2 a 5 anos de idade.

A inclusão das porções em questionários de freqüência alimentar é um assunto divergente, porém sabe-se que, quando presente, o tamanho da porção em um QFA deve estar adequado à população de estudo, como nos trabalhos de Stein et al. ${ }^{11} \mathrm{e}$ Basch et al. ${ }^{22}$. A não adaptação do tamanho da porção ao grupo estudado pode implicar em resultados sub ou superestimados ${ }^{11,22}$.

No QFAC, a porção média ou mediana mostrou-se apropriada, pois refletiu adequadamente a quantidade consumida pelas crianças e ainda facilitou o preenchimento do instrumento.

O estabelecimento de várias categorias de respostas em unidades de tempo no QFAC teve como principal objetivo fornecer ao participante a maior gama de opções, para que pudesse informar com exatidão a freqüência de consumo das crianças. As categorias existentes no QFAC foram definidas com base em estudo anterior ${ }^{13}$, considerando a facilidade de compreensão pelo entrevistado e evitando a sobreposição das categorias definidas. É discutível, dependendo da escolaridade dos entrevistados, a disponibilização de categorias de freqüência variáveis, pois isto pode influenciar a qualidade das respostas e, ao mesmo tempo, dificultar o preenchimento do instrumento. Por este motivo, no QFAC optou-se pela inclusão das mesmas categorias de resposta para todos os alimentos.

O QFAC deve ser um estímulo para o aprimoramento e desenvolvimento de novos instrumentos para avaliação do consumo alimentar de crianças, na medida em que, ao detalhar todas as etapas para o seu desenvolvimento, instrumentaliza os pesquisadores para a construção de QFA para os demais grupos da população.

\section{Referências}

1. Rockett HRH, Colditz GA. Assessing diets of children and adolescents. Am J Clin Nutr 1997; 65(Suppl): 1116S-22S.

2. Willett WC. Nutritional Epidemiology. New York, NY: Oxford University Press; 1998.

3. Serra Majem L. Evaluación del consumo de alimentos en poblaciones. Encuestas alimentarias. In: Serra Majem L et al. Nutrición y Salud Pública: métodos, bases científicas y aplicaciones. España: Masson; 1995. p. 90-106.

4. Persson LÅ, Carlgren G. Measuring children's diet: evaluation of dietary assessment techniques in infancy and childhood. Int J Epidemiol 1984; 13: 50617.

5. Willett WC, Sampson S, Stampfer MJ, Rosner BL, Bain $\mathrm{CH}$, Witschi JC. Reproducibility and validity of semiquantitative food-frequency questionnaire. Am J Epidemiol 1985; 122: 51-65.

6. Zulkifli SN, Yu SM. The food frequency method for dietary assessment. J Am Diet Assoc 1992; 92: 618-5.

7. Jiménez LG, Martín-Moreno JM. Cuestionario de frecuencia de consumo alimentario. In: Serra Majem L. Nutrición y Salud Pública: métodos, bases científicas y aplicaciones. España: Masson; 1995. p. 90-106.
8. Hammond J, Nelson M, Chinn S, Rona RJ. Validation of a food frequency questionnaire for assessing dietary intake in a study of coronary disease risk factors in children. Eur J Clin Nutr 1993; 47: 242-50.

9. Jenner DA, Neylon K, Croft S, Beilin LJ, Vandongen RA. A comparison of methods of dietary assessment in Australian children aged 11-12 years. Eur J Clin Nutr 1989; 43: 663-73.

10. Treiber FA, Leonard SB, Frank G, Musante L, Davis H, Strong WB, Levy M. Dietary assessment instruments for preschool children: reliability of parental responses to the 24 hour recall and food frequency questionnaire. J Am Diet Assoc 1990; 90: 814-20.

11. Stein AD, Shea S, Basch CE, Contento IR, Zybert P. Consistency of the Willett semiquantitative food frequency questionnaire and 24-hour dietary recalls in estimating nutrient intakes of preschool children. Am J Epidemiol 1992; 135: 667-77.

12. Furlan-Viebig, Pastor-Valero. Desenvolvimento de um questionário de freqüência alimentar para o estudo de dieta e doenças não trasnmissíveis. Rev Saúde Pública 2004; 38(4): 581-4.

13. Slater B. Desenvolvimento e validação de um questionário semi-quantitativo de freqüência alimentar para adolescentes [tese de doutorado]. São Paulo: Faculdade de Saúde Pública da USP; 2001. 
14. Monteiro CA, Conde WL. Tendência secular da desnutrição e da obesidade na infância na cidade de São Paulo (1974-1996). Rev Saúde Pública 2000; 34(6 Supl.): 52-61.

15. Instituto Brasileiro de Geografia e Estatística (IBGE). Estudo nacional de despesa familiar - ENDEF: tabela de composição de alimentos. Rio de Janeiro: IBGE; 1996.

16. Philippi ST. Tabela de composição de alimentos: suporte para decisão nutricional. Brasília, ANVISA; 2001.

17. Philippi ST, Szarfarc SC, Latterza AR. Virtual Nutri [software]. Versão 1.0 for Windows. Departamento de Nutrição/Faculdade de Saúde Pública/Universidade de São Paulo; 1996.

18. Block G, Dresser CM, Hartman AM, Carroll MD. Nutrient sources in the American diet: quantitative data from the NHANES II survey. I. Vitamins and minerals. Am J Epidemiol 1985; 122: 13-26.

19. Howe GR, Harisson L, Jain M. A short diet history for assessing dietary exposure to n-nitrosamines in epidemiologic studies. Am J Epidemiol 1986; 124: 595601 .
20. Overvad K, Tjonneland A, Haraldsdottir J, Ewertz M, Jensen OM. Development of a semiquantitative food frequency questionnaire to assess food, energy and nutrient intake in Denmark. Int J Epidemiol 1991; 20: 900-5.

21. Eck LH, Klesges RC, Hanson CL, White J. Reporting retrospective dietary intake by food frequency questionnaire in a pediatric population. J Am Diet Assoc 1991; 91: 606-8.

22. Basch CE, Shea S, Zybert P. The reproducibility of data from a food frequency questionnaire among lowincome Latina mothers and their children. Am J Public Health 1994; 84: 861-4.

23. Philippi ST, Latterza AR, Cruz ATR, Ribeiro LC. A pirâmide alimentar adaptada: guia para escolha dos alimentos. Rev Nutr 1999; 12: 65-80.

24. Galeazzi MAM, Domene SMA, Sichieri R. Estudo multicêntrico sobre consumo alimentar. Cadernos de Debate 1997 (vol. esp.).

recebido em: 13/08/2004

versão final apresentada em: 04/11/2004

aprovado em: 17/11/2004 